\title{
Chers collègues, cher(e)s ami(e)s,
}

\section{Brunaud}

(C) Lavoisier SAS 2018

Après trois années consécutives dans le sud-est de la France, cette année la Lorraine a été choisie pour accueillir le prochain rendez-vous annuel du congrès de la SOFFCO.MM à Nancy. Cette réunion rencontre un succès croissant, réunissant les différents acteurs de la prise en charge des patients obèses (chirurgiens, médecins nutritionistes, psychiatres, diététiciennes, médecins réadaptateurs, infirmiers, kinésithérapeutes, etc.).

Nous espérons vous accueillir très nombreux du 24 au 26 mai 2018 à Nancy, capitale du duché de Lorraine, célèbre pour sa place Stanislas, du nom du dernier duc de Lorraine, qui fait partie d'un ensemble du XVIII ${ }^{e}$ siècle classé au patrimoine mondial de l'Unesco. Nancy compte également de nombreux édifices classés aux monuments historiques et est l'un des foyers de l'Art nouveau grâce à l'École de Nancy. Vous pourrez découvrir ses multiples facettes historiques, architecturales, culturelles...

Le Centre Prouvé - Grand Nancy Congrès \& Evènements est un nouvel espace situé à distance de marche de la gare et du centre ville (www.grandnancy-congresetevenements. com). Le comité d'organisation rassemblera, selon l'esprit de la SOFFCO.MM, des acteurs du secteur privé et du secteur public. Comme nous essayons de le faire en pratique quotidienne dans l'unité multidisciplinaire de la chirurgie de l'obésité du CHU de Nancy, nous avons voulu que l'orientation du congrès se fasse autour de la multidisciplinarité nécessaire à une prise en charge optimale des patients obèses, que ce soit avant, pendant, ou après la chirurgie.

Les sociétés invitées à participer aux discussions seront l'AFERO, la SFNEP, les CSO et nos voisins belges avec la BeSOMS (Belgian Section of Obesity \& Metabolic Surgery). Nous avons aussi voulu faire une place importante aux jeunes chirurgien(ne)s (SOFFCO « jeunes ») et à l'innovation chirurgicale (simulateurs, robotique, etc.) avec des sessions dédiées ainsi qu'à tous nos partenaires de soins dont l'implication dans la prise en charge de l'obésité est croissante. Une conférence Delphi sur le suivi nutritionnel sera organisée. Deux sessions spécifiques seront aussi validantes pour le DPC. Enfin, le comité d'organisation a tenu spécifiquement à ce que ce congrès soit aussi un lieu de rencontres essentiel et fructueux avec nos partenaires de l'industrie.

Compte tenu du parc hôtelier disponible à cette période, nous vous incitons à réserver rapidement votre hébergement en profitant des tarifs préférentiels.

Le comité d'organisation et moi-même vous souhaitons un très enrichissant congrès et un agréable séjour.

A très bientôt à Nancy.

L. Brunaud $(\square)$

Président du Congrès SOFFCO.MM 2018

Service Chirurgie Digestive, Hépato-bilaire,

Endocrinienne et Cancérologique, UMCO, Université de Lorraine,

CHU Nancy, Hopital Brabois adultes, 11 allée du morvan,

54511 Vandoeuvre les Nancy, France

e-mail : 1.brunaud@chru-nancy.fr 